\title{
Inspiratory vocal cord closure in COPD
}

\author{
To the Editor:
}

During normal inspiration, vocal cord movements are limited, although mild abduction (widening) can occur [1]. Inspiratory closure is characteristic of vocal cord dysfunction (VCD), a condition that can cause refractory breathlessness $[1,2]$. VCD may occur in otherwise healthy individuals and $20-40 \%$ of stable asthma patients [3], however, abnormal inspiratory vocal cord activities and putative VCD have not been investigated in other obstructive lung diseases. It is therefore of interest to determine whether inspiratory closure occurs in COPD.

We hypothesised that inspiratory closure may occur frequently in COPD as well as during acute exacerbations of COPD and dynamic computed tomography (CT) of the larynx was used to evaluate three groups. Healthy individuals and patients with COPD who had participated in studies examining excessive dynamic airway collapse (EDAC) [4] were assessed. A control group consisted of healthy older volunteers without respiratory symptoms $(n=40)$. The stable COPD group was comprised of patients in the original cohort [4] as well as additional outpatients with breathlessness on exertion $(n=76)$. No patients had acute exacerbations of COPD in the preceding 3 months. The third group consisted of consecutive patients hospitalised with acute exacerbation of $\operatorname{COPD}(n=61)$, and in this group pulmonary function testing was performed after recovery from the exacerbation. Individuals with acute exacerbation of COPD underwent dynamic CT of the larynx as soon as possible after admission (mean \pm SD $12 \pm 7 \mathrm{~h}$ ). COPD was diagnosed by specialist pulmonologists and verified by spirometry, with airflow limitation classified according to the Global Initiative for Chronic Obstructive Lung Disease [5]. No patients had a history or other evidence of asthma. Studies were done at Monash Medical Centre, Melbourne, Australia and all studies were approved by Monash Health Human Research Ethics Committee. Patients gave informed consent.

Although the diagnostic gold standard for inspiratory closure is nasal laryngoscopy, this technique has limitations $[6,7]$ and we have developed an alternative approach: dynamic CT of the larynx. The method can be used to diagnose inspiratory closure and presumptive VCD in chronic asthma [6] and we have recently reported studies in acute asthma exacerbations [8]. In brief, measurements of vocal cord diameter are made at $0.35 \mathrm{~s}$ intervals, normalised to tracheal measurements and integrated into a diagnostic algorithm. Patient curves covering the breathing cycle are generated and compared to standard curves and inspiratory closure can be detected if the patient curve is below the lower limit of normal curve during inspiration (or during both inspiration and expiration but not expiration only). The method has been shown to have good specificity and to be equivalent to laryngoscopy in stable disease $[3,6]$. In the current studies, patients without and with inspiratory closure were compared using Fisher's exact test (categorical data) and appropriate non-parametric tests (continuous data).

Patient characteristics are shown in the table 1 . No healthy volunteers had abnormal vocal cord movement. However, inspiratory closure was detected in the stable outpatient COPD group in 21/76 patients $(27.6 \%)$ and the acute exacerbation of COPD group in $19 / 61$ patients $(31.1 \%)$. Within the stable COPD group, demographic variables (age, sex, body mass index, spirometry including bronchodilator response and diffusing capacity) did not differ between patients with inspiratory closure detected and not detected. Age, sex, smoking history, body mass index, forced expiratory volume in $1 \mathrm{~s}$, bronchodilator response and diffusing capacity were similar, as were exacerbation characteristics, including BAP65 [9] class and length of stay. In both COPD groups, inhaled medications did not significantly differ between patients with or without inspiratory closure.

The current studies therefore did not detect inspiratory closure in healthy individuals, but the abnormality was noted in approximately one quarter of patients with COPD. In a separate group with acute exacerbation of COPD the prevalence was similar. These preliminary findings indicate that the 
TABLE 1 Demographic characteristics of subjects included in studies

\begin{tabular}{|c|c|c|c|c|}
\hline & $\begin{array}{l}\text { Healthy individuals } \\
\qquad(\mathrm{n}=40)\end{array}$ & $\begin{array}{c}\text { Stable COPD } \\
(n=76)\end{array}$ & $\begin{array}{c}\text { Acute } \\
\text { exacerbations } \\
\text { of COPD }(n=61)\end{array}$ & $\begin{array}{l}\mathrm{p} \text {-value for } \\
\text { group } \\
\text { comparison }\end{array}$ \\
\hline Age years & $61.6 \pm 12.0$ & $69.3 \pm 10.2$ & $71.5 \pm 11.1$ & 0.70 \\
\hline Sex (females/males) & $25 / 15$ & $33 / 43$ & $23 / 38$ & 0.35 \\
\hline Body mass index $\left(\mathrm{kg} \cdot \mathrm{m}^{-2}\right)$ & $29.5 \pm 5.7$ & $28.3 \pm 7.4$ & $24.9 \pm 4.9$ & 0.19 \\
\hline Smoking pack-years & $8.6 \pm 13.6$ & $41.1 \pm 25.6$ & $59.1 \pm 51.9$ & 0.027 \\
\hline \multicolumn{5}{|l|}{ Lung function } \\
\hline $\begin{array}{l}\mathrm{FEV}_{1} \text { post-bronchodilator \% } \\
\text { pred }\end{array}$ & $100.9 \pm 16.7$ & $55.6 \pm 23.3$ & $48.2 \pm 19.8$ & 0.062 \\
\hline $\mathrm{FEV}_{1} / \mathrm{FVC} \%$ & $76.1 \pm 7.6$ & $46.2 \pm 13.3$ & $48.8 \pm 15.0$ & 0.31 \\
\hline Bronchodilator response $\%$ & $3.5 \pm 3.7$ & $9.0 \pm 9.4$ & $6.2 \pm 7.9$ & 0.21 \\
\hline$T_{\text {LCO }} \%$ pred & $89.6 \pm 13.6$ & $58.9 \pm 21.7$ & $49.9 \pm 21.3$ & 0.032 \\
\hline \multicolumn{5}{|l|}{ Inhaled treatment(s) } \\
\hline Inhaled corticosteroids & None & $14(18.4 \%)$ & $43(70 \%)$ & $<0.0001$ \\
\hline Long-acting $\beta$-agonist & None & $59(77.6 \%)$ & $43(70 \%)$ & 0.43 \\
\hline $\begin{array}{l}\text { Long-acting muscarinic } \\
\text { antagonist }\end{array}$ & None & $58(76.3 \%)$ & $38(62 \%)$ & 0.09 \\
\hline $\begin{array}{l}\text { Inspiratory vocal cord } \\
\text { closure }\end{array}$ & None & $21(27.6 \%)$ & $19(31.1 \%)$ & 0.71 \\
\hline Expiratory vocal cord closure & None & $22(28.9 \%)$ & $17(27.9 \%)$ & 1.0 \\
\hline
\end{tabular}

Values are presented as mean \pm SD or $n(\%)$, unless otherwise stated. $\mathrm{p}$ values from ANOVA, or Fisher's exact test as appropriate. $\mathrm{FEV}_{1}$ : forced expiratory volume in $1 \mathrm{~s}$; FVC: forced vital capacity; $T_{\text {LCo: }}$ diffusing/transfer capacity for carbon monoxide.

quintessential inspiratory abnormality of VCD may be common in COPD and that VCD may be happening "under our noses" [5]. Whether patients had "true" VCD requires further study, considering the need to have abnormal inspiratory vocal cord movement occurring with commensurate symptoms in an applicable clinical context. Our findings also infer that VCD may compound symptoms, at least in some individuals with COPD; this aspect also requires future study.

The findings are not entirely surprising. Our previous studies in asthma suggested that airway obstruction with associated dysfunctional breathing may cause laryngeal activation leading to VCD in many patients [3]. This pathophysiology may also be applicable in COPD given the high prevalence of dysfunctional breathing in COPD [10]. Other explanations are possible. For example, functional expiratory laryngeal changes have been reported in COPD [11] with expiratory laryngeal closure during exercise, which may be a plausible physiological mechanism to generate positive end-expiratory pressure. Although inspiratory closure is generally considered abnormal [12], inspiratory closure may be part of a compensatory strategy in COPD that remains to be elucidated.

Why has inspiratory closure of the vocal cords and putative VCD not been suspected in COPD? There are several possibilities, but a likely reason is that patients with COPD have impaired respiratory function (as part of the diagnosis) and therefore an attributable cause for their breathlessness. Additionally, COPD symptoms are often not resolved by treatment. Asthma, in contrast, is characterised by (mostly) normal lung function leading to early suspicion of VCD if breathlessness is unresponsive to treatments. Overall, our findings emphasise the importance of the "middle airway", a key part of the airway long ignored [7] that may play a cardinal role in the generation of more severe symptoms in asthma and COPD.

There are important aspects to consider in the interpretation of this preliminary data. Care was taken to exclude asthma patients, but it is possible that a small number may have been included. Careful repeat review of patients with COPD found no evidence of asthma. All were smokers ( $>40$ pack-years in both COPD groups), had impaired pulmonary function and had been diagnosed and treated for COPD by a respiratory specialist. The COPD groups were also older than the healthy controls. Since age has not been linked to VCD, this factor appears unlikely to explain our findings, particularly since there was a large overlap in age and many healthy individuals were older than persons with COPD and VCD. Finally, initial validation of the algorithm used to detect inspiratory closure was conducted in asthma. Similar validation in COPD has not been done but it appears unlikely that application of the technique in COPD is not valid. 
In summary, we report inspiratory vocal cord closure coexisting with COPD, which may amplify breathlessness, and blunt pharmacological and other treatments. VCD should be considered as a comorbidity not only in asthma, but also in COPD, and further investigation is merited.

Paul Leong ${ }^{1,2,6}$, Laurence E. Ruane ${ }^{1,2,6}$, Debra Phyland ${ }^{3}$, Joo Koh ${ }^{3}$, Martin I. MacDonald ${ }^{1,2}$, Malcom Baxter ${ }^{3}$, Kenneth K. Lau ${ }^{4}$, Kais Hamza ${ }^{5}$ and Philip G. Bardin ${ }^{1,2}$

${ }^{1}$ Monash Lung and Sleep, Monash Health and Monash University, Clayton, Australia. ${ }^{2}$ Hudson Institute, Clayton, Australia. ${ }^{3}$ Dept of Otolaryngology, Head and Neck Surgery, Monash Health, Clayton, Australia. ${ }^{4}$ Diagnostic Imaging, Monash University and Monash Health, Clayton, Australia. ${ }^{5}$ Dept of Mathematical Sciences, Monash University, Clayton, Australia. ${ }^{6}$ Equal contribution.

Correspondence: Paul Leong, Monash Lung and Sleep, 246 Clayton Rd, Clayton, Victoria, 3168, Australia. E-mail: paul. leong@monash.edu

Received: 23 July 2019 | Accepted after revision: 22 Jan 2020

Acknowledgements: The authors would like to thank Paul Finlay and Kathy Low for their contribution to this study.

Conflict of interest: None declared.

Support statement: P. Leong is supported by the Australian National Health and Medical Research Council (Postgraduate Scholarship) and the Royal Australasian College of Physicians (Dixon Award) outside of the present work. Studies were funded by an unrestricted support grant from Monash Lung and Sleep Institute. Views expressed do not necessarily reflect those of the funding bodies. Funding information for this article has been deposited with the Crossref Funder Registry.

\section{References}

1 Hull JH, Backer V, Gibson PG, et al. Laryngeal dysfunction: assessment and management for the clinician. Am J Respir Crit Care Med 2016; 194: 1062-1072.

2 Christopher KL, Wood RP, Eckert RC, et al. Vocal-cord dysfunction presenting as asthma. N Engl J Med 1983; 308: 1566-1570.

3 Low K, Ruane L, Uddin N, et al. Abnormal vocal cord movement in patients with and without airway obstruction and asthma symptoms. Clin Exp Allergy 2017; 47: 200-207.

4 Leong P, Tran A, Rangaswamy J, et al. Expiratory central airway collapse in stable COPD and during exacerbations. Respir Res 2017; 18: 163.

5 Global Initiative for Chronic Obstructive Lung Disease (GOLD). Global Strategy for the Diagnosis, Management and Prevention of COPD, 2019. https://goldcopd.org/gold-reports/

6 Low K, Lau KK, Holmes P, et al. Abnormal vocal cord function in difficult-to-treat asthma. Am J Respir Crit Care Med 2011; 184: 50-56.

7 Bardin PG, Johnston SL, Hamilton G. Middle airway obstruction-it may be happening under our noses. Thorax 2013; 68: 396-398.

8 Daley CP, Ruane LE, Leong P, et al. Vocal cord dysfunction in patients hospitalised with symptoms of acute asthma exacerbation. Am J Respir Crit Care Med 2019; 200: 782-785.

9 Shorr AF, Sun X, Johannes RS, et al. Validation of a novel risk score for severity of illness in acute exacerbations of COPD. Chest 2011; 140: 1177-1183.

10 Law N, Ruane LE, Low K, et al. Dysfunctional breathing is more frequent in chronic obstructive pulmonary disease than in asthma and in health. Respir Physiol Neurobiol 2018; 247: 20-23.

11 Baz M, Haji GS, Menzies-Gow A, et al. Dynamic laryngeal narrowing during exercise: a mechanism for generating intrinsic PEEP in COPD? Thorax 2015; 70: 251-257.

12 Morris MJ, Christopher KL. Diagnostic criteria for the classification of vocal cord dysfunction. Chest 2010; 138: $1213-1223$. 